\title{
Anabases
}

ANABASES Traditions et réceptions de l'Antiquité

$16 \mid 2012$

Varia

\section{Un inédit de Michel Foucault : «La Parrêsia ». Note de présentation}

Henri-Paul Fruchaud et Jean-François Bert

\section{(2) OpenEdition}

\section{Journals}

Édition électronique

URL : https://journals.openedition.org/anabases/3956

DOI : 10.4000/anabases.3956

ISSN : 2256-9421

Éditeur

E.R.A.S.M.E.

\section{Édition imprimée}

Date de publication : 1 octobre 2012

Pagination : 149-156

ISSN : 1774-4296

\section{Référence électronique}

Henri-Paul Fruchaud et Jean-François Bert, « Un inédit de Michel Foucault : «La Parrêsia ». Note de présentation », Anabases [En ligne], 16 | 2012, mis en ligne le 01 octobre 2015, consulté le 21 septembre 2021. URL : http://journals.openedition.org/anabases/3956 ; DOI : https://doi.org/ 10.4000/anabases.3956 
Anabases 16 (2012), p. 149-156.

\section{Un inédit de Michel Foucault : "La Parrêsia" Note de présentation}

Henri-Paul Fruchaud, JeAN-FranÇOIS BerT

$\mathrm{C}_{\text {EST À l'invitation d'Henri Joly }}{ }^{1}$, spécialiste de la philosophie antique, que Michel Foucault prononce au mois de mai 1982 à l'université de Grenoble une conférence consacrée à la parrêsia, peu de temps après la fin du cours au Collège de France de l'année 1982, dans lequel cette notion apparait pour la première fois dans ses travaux. Henri Joly connaissait Foucault depuis son passage à Clermont Ferrand, et comme le précise Pascal Engel : «Le spécialiste de Platon qu'était Joly s'intéressait au "retour aux Grecs" de Foucault et ce dernier avait accepté de venir donner un exposé. Nous allâmes ensemble le chercher à la gare, en l'attendant à la sortie principale, mais là point de Foucault. La gare de Grenoble a une seconde sortie, quasi clandestine, qu'on prend rarement. Michel Foucault trouva le moyen de passer par là et nous eûmes la surprise de l'entendre nous héler derrière nous. Il était, comme le dit une page célèbre de L'Archéologie du savoir, "ressurgi ailleurs" et "en train de nous narguer ${ }^{2 "}$. [...] Il avait exigé qu'on ne publicisât pas sa conférence, afin que la discussion puisse avoir lieu en

1 Henri Joly (1927-1988) est notamment l'auteur d'un ouvrage consacré à Platon : Le Renversement platonicien. Logos, épistémè, polis, Paris, Vrin, 1974.

2 Ce passage se trouve dans le dialogue fictif entre Foucault et son lecteur par lequel se termine l'Introduction de L'Archéologie du savoir (Paris, Gallimard, 1969, p. 28). 
petit comité. Mais quand nous pénétrâmes dans la salle, plus d'une centaine de personnes nous attendaient et comme jadis au Collège la conférence tourna au spectacle ${ }^{3}$. "

Cette conférence, dont un enregistrement a été conservé, était restée jusqu'à ce jour inédite ${ }^{4}$.

Michel Foucault a consacré une grande partie de ses derniers travaux à la notion de parrêsia ${ }^{5}$, que l'on traduit habituellement par " franc-parler ». Elle apparaît dans le cours au Collège de France de 1982, L’herméneutique du sujet, et sera le thème principal des cours de 1983, Le gouvernement de soi et des autres, et de 1984, Le courage de la véritét . Il consacre également à la parrêsia un cycle de six conférences prononcé à l'université de Berkeley en octobre et novembre $1983^{7}$.

Cette notion apparaît dans le cadre d'une réflexion de Foucault sur les rapports de la vérité et du sujet. Après avoir étudié ce qu'il appelle les « formes alèthurgiques » - les pratiques discursives qui ont permis de constituer, comme objet de savoir possible, le sujet parlant, le sujet travaillant, le sujet vivant ${ }^{8}-$ Foucault renverse la perspective et oriente ses recherches vers la constitution du sujet pour lui-même. On sait que pour lui, le sujet fondateur et anhistorique de la philosophie classique, de Descartes à la phénoménologie et à l'existentialisme, n'existe pas. Au contraire, celui-ci a une genèse, une histoire, à travers lesquelles il se constitue et se transforme. En étudiant cette " histoire de la subjectivité », entendue au sens de "la manière dont le sujet fait l'expérience de

3 Pascal EnGEL, "Michel Foucault : vérité, connaissance et éthique ", in Foucault, Paris, Éditions de l'Herne, 2011, p. 324. Pascal Engel date la conférence du printemps 1983. Nous avons retenu la date de mai 1982, proposée par Daniel Defert d'après les archives de Michel Foucault. Elle paraît d'ailleurs plus vraisemblable, dans la mesure où l'analyse de la parrêsia dans la conférence est encore très proche des cours du début de l'année 1982.

$4 \quad$ Un texte de Michel Foucault a été publié en 1983 dans le n 3 des Recherches sur la philosophie et le langage. La philosophie dans sa langue, dirigé par Henri Joly, mais il s'agit d'un texte intitulé : "Rêver de ses plaisirs. Sur l'onirocritique d'Artémidore ".

5 Les translitérations parrhêsia ou parrhèsia sont les plus conformes au grec ancien, mais pour la transcription de la conférence de Grenoble, nous nous sommes conformés à la solution retenue pour l'édition des derniers cours de Foucault et la plus couramment utilisée dans les études foucaldiennes.

6 Michel Foucault, L'herméneutique du sujet, Paris, Gallimard/Le Seuil, 2001 ; Le gouvernement de soi et des autres, Paris, Gallimard/Le Seuil, 2008 ; Le courage de la vérité. Le gouvernement de soi et des autres II, Paris, Gallimard/Le Seuil, 2009. Pour une présentation synthétique de la parrêsia, voir Frédéric Gros, "La parrhêsia chez Foucault (1982-1984)", in Foucault. Le courage de la vérité, Paris, PUF, 2002, p. 155-165, et Michel Foucault, Paris, PUF, 1998, p. 110-123.

7 Ces conférences ne sont pas traduites en français. Une transcription, parfois abusivement remaniée par l'éditeur, a été publiée sous le titre de Fearless Speech, Semiotext(e), Los Angeles, 2001.

8 Michel Foucault, Le courage de la vérité, édition citée, p. 5. 
lui-même dans un jeu de vérité où il a rapport à soi ${ }^{9}$ ", Foucault va s'intéresser en premier lieu au christianisme primitif à propos de cette forme de dire-vrai qu'est l'aveu, puis, remontant le temps, à la philosophie antique, et plus particulièrement à la philosophie gréco-romaine de l'époque impériale.

C'est dans son cours du 10 février 1982 qu'il fait pour la première fois mention de la parrêsia, à laquelle il consacrera la totalité du cours du 10 mars. Après avoir caractérisé la philosophie antique par le souci de soi, l'epimeleia heautou, il rappelle que c'est dans le souci de soi, par la conversion à soi à travers l'acquisition de connaissances vraies et la pratique d'exercices spirituels, que le sujet de l'Antiquité se constitue comme sujet éthique, capable de se gouverner lui-même et de gouverner les autres. Mais pour pratiquer ce souci de soi, le sujet a besoin d'un autre : un maître, un directeur. Cet autre pouvant être, comme Épictète, un professeur exerçant au sein d'une école de philosophie, un ami ou un parent, comme Sénèque vis-à-vis de Sérénus ou de Lucilius, ou encore un conseiller privé comme en avaient parfois de grands personnages romains. Or, pour jouer ce rôle, l'autre a toujours besoin d'être doté de parrêsia, notion dont Foucault, avant d'en affiner le sens à travers l'analyse des textes, donne une première définition : "Parrhêsia, étymologiquement, c'est le fait de tout dire (franchise, ouverture de parole, ouverture d'esprit, ouverture de langage, liberté de parole). Les Latins traduisent en général parrhêsia par libertas. C'est l'ouverture qui fait qu'on dit, qu'on dit ce qu'on a à dire, qu'on dit ce qu'on a envie de dire, qu'on dit ce qu'on pense pouvoir dire, parce que c'est nécessaire, parce que c'est utile, parce que c'est vrai ${ }^{10}$. "

La parrêsia du maître est à la fois une technê, une technique, et un ethos, une manière d'être. En tant que technique, elle lui permet d'aider le disciple à se connaître et à se doter des vérités dont il a besoin pour faire face aux événements de la vie et pour vivre une vie véritablement philosophique. Mais elle ne peut réussir comme technique si elle n'est pas également une manière d'être par laquelle le maître manifeste qu'il est lui-même comme ce qu'il dit, faisant en sorte que ce qu'il dit soit immédiatement reçu comme vrai par le disciple.

Dans les cours des deux années suivantes, Foucault élargira et approfondira la notion de parrêsia, et ceci dans deux directions. La première est celle de la parrêsia politique, dans le cadre de la démocratie grecque, plus particulièrement athénienne, et à la cour du prince.

Dans le cadre démocratique, la parrêsia est un droit politique qui permet à l'homme politique influent (l'exemple sera Périclès), de participer activement au gouvernement de la cité et d'emporter l'adhésion de ses concitoyens par la franchise de son discours. Foucault s'intéressera à l'histoire mythique de cette parrêsia démocratique en analysant quelques tragédies d'Euripide, en particulier Ion, qu'il interprétera comme

9 Maurice Florence (pseudonyme de Michel Foucault), "Foucault ", in Dits et écrits, $n^{\circ}$ 345, t. II, Paris, Gallimard "Quarto », 2001, p. 1452.

10 Michel Foucault, L’herméneutique du sujet, édition citée, p. 348. 
l'histoire de la vérité abandonnée par les dieux et reprise par les hommes, transférée symboliquement du temple de Delphes à Athènes, où elle sera l'un des fondements de la démocratie. À cette bonne parrêsia démocratique s'oppose une mauvaise parrêsia, analysée notamment à travers la critique du régime démocratique formulée par Platon et certains orateurs ou hommes politiques contemporains de la crise de la démocratie athénienne ; c'est celle de la cité où chacun dit et fait ce qu'il a envie de dire et de faire, ou bien dans laquelle seuls sont écoutés les orateurs qui flattent le peuple et ne lui disent que ce qu'il a envie d'entendre. La parrêsia à la cour du prince est aussi longuement analysée par Foucault à travers l'exemple de Platon à la cour de Denys de Syracuse. Avec la montée des monarchies hellénistiques, cette forme de la parrêsia politique va devenir dominante; le bon prince est celui qui accepte que ses conseillers pratiquent la parrêsia à son égard. Ses investigations sur la parrêsia politique conduiront Foucault à associer très étroitement la notion de risque à la pratique de la parrêsia : celui qui dit la vérité, qu'il soit homme politique, orateur ou conseiller du prince, est quelqu'un qui prend un risque, pouvant aller jusqu'à celui de perdre la vie, pour dire la vérité, et c'est précisément à cela qu'on reconnaît qu'il est un parrèsiaste.

La seconde direction est celle de la parrêsia éthique. Foucault, en effet, sera conduit à opposer à la conception moderne de la vérité - qui est la nôtre depuis Descartes pour laquelle le sujet accède à la vérité par la seule connaissance, dans le respect de règles de forme et d'objectivité, une conception antique pour laquelle l'accès à la vérité requiert du sujet qu'il se transforme lui-même, qu'il devienne un sujet éthique de la vérité $^{11}$. Ce sont trois principaux moments qu'analyse Foucault. Le moment socratique, lorsque la parrêsia commence à se détacher de la politique pour rentrer dans le cadre des relations interpersonnelles; Socrate, parce qu'il en a reçu du dieu la mission, est celui qui éprouve les âmes, les aide à découvrir la vérité pour donner forme à leur existence. Le moment cynique, où dans la parrêsia se rejoignent et coïncident exactement un dire-vrai souvent brutal et provocateur et un style de vie absolument dépourvu d'attaches et complètement libre. Enfin le moment de la philosophie gréco-romaine des deux premiers siècles de l'empire, étudié dès 1982 dans L'herméneutique du sujet et qui sera également le thème central de la conférence de Grenoble. Pour accéder à la vie philosophique, devenue une affaire complètement privée, on a absolument besoin de la parrêsia de l'autre ; il s'agit maintenant de savoir comment reconnaître cet autre et quels rapports établir avec lui ${ }^{12}$.

11 Michel Foucault, L’herméneutique du sujet, édition citée, p. 19.

12 La parrêsia politique est principalement étudiée dans le cours de 1983, Le gouvernement de soi et des autres. Le basculement vers la parrêsia éthique est abordé à la fin de ce cours. Le cours de 1984, Le courage de la vérité, porte sur les moments socratique et cynique; le moment gréco-romain avait été étudié en premier dans L'herméneutique du sujet. Dans les conférences de Berkeley de fin 1983, Foucault retrace, sous une forme plus condensée, l'évolution de la notion de parrêsia, en suivant l'ordre chronologique. 
Si elle est proche du cours de 1982 par la période étudiée et les textes analysés, la conférence de Grenoble présente quelques particularités qui contribuent à la rendre très intéressante. Au début de la conférence, Foucault indique qu'il vient présenter un travail en train de se faire : "C'est, dit-il, des matériaux, c'est des références à des textes, des indications; l'exposé que je vais vous faire est donc lacunaire... ». On y voit s'esquisser un certain nombre de pistes qui seront explorées et approfondies dans les deux dernières années de cours. Beaucoup de développements futurs sont déjà en germe dès le printemps 1982.

D’abord du côté de la parrêsia politique, avec les textes tirés de quatre tragédies d'Euripide (Ion, Hippolyte, Les Phéniciennes, Les Bacchantes) sur lesquelles Foucault reviendra longuement l'année suivante. La mauvaise parrêsia politique fait également une apparition fugitive à travers le texte de La République sur les caractères du régime démocratique et la parrêsia du conseiller de prince à travers la description du royaume de Cyrus dans Les Lois et le discours À Nicoclès d'Isocrate. Ensuite du côté du moment socratique de la parrêsia éthique, lorsque Foucault commente le passage du Gorgias sur la pierre de touche, le basanos, dont l'âme a besoin pour accéder à la vérité. Seule la parrêsia cynique, qui prendra tant d'importance en 1984, est encore absente ; la " violence diatribique " sera même opposée à la transparence du discours parrèsiastique. Foucault verra tout autrement la parrêsia cynique dans les cours de 1983 et surtout de 1984 .

Plus, peut-être, que dans son cours au Collège de France, on voit ici Foucault au travail. Probablement parce qu'il s'adresse à des spécialistes de la pensée antique, il adopte un mode d'exposition des textes un peu différent, plus proche du mouvement de la recherche. Il ne donne au début de la conférence qu'une définition très succincte de la parrêsia, qualifiée de " tout-dire » ou "d'obligation de dire ». C'est au final le seul parcours à travers les textes commentés qui va lui permettre, progressivement, d'en faire apparaitre le véritable sens. De là vient sans doute une tension qui court tout au long de la conférence, et qui se résorbe et s'apaise lorsque, tout à la fin, Foucault commente le texte de la Lettre 75 de Sénèque à Lucilius, où la parrêsia semble enfin rejoindre ce qu'elle est : l'implication et la manifestation, dans une transparence totale, de celui qui parle dans la vérité de ce qu'il dit.

Concernant le deuxième et le troisième volume de l' Histoire de la sexualité, Pierre Hadot avait pu affirmer que si Foucault était un bon historien des faits sociaux et des idées, il n’avait jamais pratiqué la philologie, ne s'était pas posé la question des problèmes liés à la tradition des textes, du déchiffrement des manuscrits, des éditions critiques, du choix des variantes textuelles ${ }^{13}$. C'est l'une des nombreuses formes qu'a

13 Pierre Надот, La philosophie comme manière de vivre, Paris, Albin Michel, 2001, p. 214-215. Maria Daraki, dans «Le voyage en Grèce de Michel Foucault » (Esprit, avril 1985, p. 55-83), multiplia elle aussi ce type de critique. 
pu prendre le "malentendu ${ }^{14}$ " constant entre Foucault et les historiens, malentendu qui franchira un nouveau cap en 1978 alors que Foucault décide de se tourner dans son cours au Collège de France vers un nouveau corpus de textes, inédit pour lui, celui des Pères de l'Église.

De L'archéologie du savoir (1969), en effet, les historiens ne semblent avoir retenu que la prose critique du philosophe et pas sa tentative de mettre en place une historiographie qui aurait pour tâche de déterminer quelle forme de relations peut être légitimement décrite entre des séries, des découpes, des limites ${ }^{15}$. Dix ans plus tard, à l'occasion d'un débat avec les historiens de la société d'histoire de 1848 (Maurice Agulhon en tête), Foucault réitère une proposition quasi identique. Une constance qui peut aujourd'hui étonner! Il s'agit, dit-il, d'élaborer une histoire des singularités et donc de " retrouver les connexions, les rencontres, les appuis, les blocages, les jeux de force, les stratégies, etc., qui ont, à un moment donné, formé ce qui ensuite va fonctionner comme évidence, universalité, nécessité ${ }^{16}$ ». Là encore, la proposition resta sans véritable effet.

1978 reste à bien des égards une date pivot pour Foucault.

Son cours sur le Gouvernement des vivants (1979-1980) qui fait une place centrale au corpus patristique (en particulier le De Paenitentia de Tertullien) va l'obliger à pratiquer pour la première fois le texte original ${ }^{17}$. Mais 1978, c'est aussi l'année où il découvre, sur la suggestion de Paul Veyne, The Making of Late Antiquity de Peter Brown ${ }^{18}$. C'est d'ailleurs en 1978 que l'historien de l'Antiquité défend, à partir des ouvrages de Foucault, la possibilité d'une histoire plus réflexive, interdisciplinaire et

14 Les textes tentant de faire le point sur ce malentendu sont nombreux, citons cependant : Jacques ReVel, "Le moment historiographique ", in Luce Giard, Michel Foucault, lire l'auvre, Grenoble, Jérôme Millon, 1992, p. 83-96 ; Arlette FARGE, "Face à l'histoire ", Magazine littéraire, $\mathrm{n}^{\circ} 207,1984$, p. 40-42, et dans une version plus critique Gérard NoIriel, "Foucault et l'histoire : les leçons d'un désenchantement ", in Penser avec, penser contre. Itinéraire d'un historien, Paris, Belin, 2003, p. 25-46.

15 L'archéologie du savoir, op. cit., p. 17-19.

16 L'Impossible prison, Recherches sur le système pénitentiaire au XIX siècle, Paris, Le Seuil, coll. "L'Univers historique ", 1980, p. 44.

17 Comme le note Philippe Chevallier dans son étude sur Michel Foucault et le christianisme : "Si Foucault revient au texte original, c'est afin que la traduction proposée lui ressemble le plus possible, dans sa syntaxe comme dans sa phonie. » Philippe CHEvallier, Michel Foucault et le christianisme, Paris, ENS Éditions, 2012, p. 200.

18 C'est encore en 1978 que le livre de K.J. Dover dont s'inspirera Foucault pour L'usage des plaisirs est publié : Greek Homosexuality, Londres, Duckworth, 1978. Un livre précédé en 1974 par Greek Popular Morality in the Time of Plato and Aristotle, lui aussi cité par Foucault. 
qui, par la mise en intrigue, doit permettre aux historiens de rompre avec les fausses continuités historiques ${ }^{19}$.

Quatre ans après, cette conférence de Grenoble montre peut-être mieux qu'ailleurs le va-et-vient constant que Foucault cherche à mettre en place entre les textes originaux, leur commentaire littéral, et les enjeux philosophiques, éthiques et surtout politiques que sa lecture sous-tend. Enjeux qui seront par la suite déplacés, reformulés. On peut voir à l'œuvre sa nouvelle attention aux textes, par exemple dans sa manière de construire des concepts à partir de termes classiques. Selon les cas, il décide de forger des néologismes plutôt que d'utiliser le terme français courant comme " aléthurgique " (dans son cours du 9 janvier 1980), " exomologétique ", " psychagogique " (dans son cours de 1982). Foucault témoigne d'une grande attention à la langue ainsi qu'à l'importance conceptuelle du terme. Il ne s'agit pas seulement pour lui de bien traduire mais de bien désigner le concept.

Son retour aux Grecs va l'obliger à changer ses manières de lire. Sa longue introduction à L'usage des plaisirs rappelle cette préoccupation importante ; lui qui n'était ni helléniste, ni latiniste : «Le danger était aussi d'aborder des documents de moi trop mal connus. Je risquais de les plier, sans trop m'en rendre compte, à des formes d'analyse ou à des modes de questionnement qui, venus d'ailleurs, ne leur convenaient guère ${ }^{20}$. " C'est pourtant cette Grèce de L'usage des plaisirs qui permit, entre autres, à Jean-Pierre Vernant de "considérer le problème de la sexualité dans le monde grec ancien ${ }^{21}$ ".

Ce «dernier Foucault ", celui des techniques de soi qui consistent pour chacun à trouver, en soi-même, la manière de se conduire et surtout de se gouverner, ne doit pas nous faire oublier que Foucault eut l'occasion, lors de son premier cours au Collège de France, de montrer sa bonne familiarité avec l'historiographie de la période hellénistique.

En effet, dans La volonté de savoir, Foucault cherche à comprendre la manière dont les formes juridiques ont eu en Grèce antique la prétention de départager le vrai du faux, passant de la vérité-défi à la vérité-savoir « liée en sa racine à la justice, à la distribution et à l'ordre ${ }^{22}$ ». Certes, la Grèce est abordée par le biais de Nietzsche -

19 Paul VeYNE, "Foucault révolutionne l'histoire ", in Comment on écrit l'histoire, Paris, Le Seuil, 1978, p. 385-429, et Les Grecs ont-ils cru à leurs mythes ?, Paris, Le Seuil, 1983.

20 Michel Foucault, L'usage des plaisirs, Paris, Gallimard, 1984, p. 14-15.

21 Voir Jean-Pierre Vernant, "Entretien inédit : "Comme une barque sur un fleuve..." ", Anabases 7 (2008), p. 17-32. Ainsi que la discussion qui suivit l'intervention de Pierre Hadot : "Réflexion sur la notion de "culture de soi" ", in Michel Foucault, Philosophe, Paris, Le Seuil, 1989, p. 270. Rappelons aussi que deux ans après les deux derniers tomes de L'Histoire de la sexualité des chercheurs français et américains se sont retrouvés pour réinterroger la question de la sexualité antique : les actes furent réunis en un volume intitulé Before Sexuality: The Construction of Erotic Experience in the Ancient Greek World (éd. Winkler Halperin, Froma Zeituin, Princeton, 1990).

22 Leçons sur la Volonté de savoir, 1970-1971, Paris, Gallimard/Le Seuil, 2011, p. 123. 
Foucault tente une généalogie de la connaissance - mais il explore aussi les textes d'historiens : ceux de Louis Gernet, Gustave Glotz et Édouard Will, mais aussi des ouvrages, alors récents, comme Le pur et l'impur dans la pensée des Grecs d'Homère à Aristote de L. Moulinier ou encore Les maîtres de vérité de Marcel Detienne.

En 1986, à l'occasion d'un numéro spécial de la revue Le Débat sur Foucault, Henri Joly chercha à faire le point sur ce « retour aux Grecs» de Foucault. Un terrain grec qui pose à l'historien de redoutables problèmes, en premier celui de la continuité et de la discontinuité. Mais Joly déplace le point de vue et préfere souligner que l'apport essentiel de Foucault se situe dans sa manière de lire les textes avec une "insistante méticulosité ", incitant les historiens à ne plus les lire comme avant : "C'est tout un pan du monde grec qui a bougé ${ }^{23}$."

Le texte de la conférence est la transcription littérale d'un enregistrement, dont la qualité sonore est assez médiocre.

On a simplement, lorsque cela paraissait indispensable, supprimé quelques interjections et répétitions et rétabli la construction de quelques phrases. Certaines références incorrectes ont également été corrigées. Les passages inaudibles et les mots ajoutés sont indiqués entre crochets.

Bien qu'elle soit en partie inaudible, sans doute parce que certains interlocuteurs étaient éloignés de la source d'enregistrement, nous avons conservé la discussion finale, notamment pour l'intérêt des échanges entre Henri Joly et Michel Foucault.

23 Henri Joly, "Retour aux Grecs : réflexions sur les "pratiques de soi" dans L'usage des plaisirs ", Le Débat 41 (septembre-novembre 1986), p. 100-120. 